\title{
Small Group Divisible Steiner Quadruple Systems
}

\author{
Artem A. Zhuravlev, Melissa S. Keranen, Donald L. Kreher \\ Department of Mathematical Sciences, \\ Michigan Technological University \\ Houghton, MI 49913-0402, USA
}

Submitted: Oct 24, 2007; Accepted: Feb 29, 2008; Published: Mar 12, 2008

Mathematics Subject Classification: 05B05

\begin{abstract}
A group divisible Steiner quadruple system, is a triple $(X, \mathcal{H}, \mathcal{B})$ where $X$ is a $v$-element set of points, $\mathcal{H}=\left\{H_{1}, H_{2}, \ldots, H_{r}\right\}$ is a partition of $X$ into holes and $\mathcal{B}$ is a collection of 4-element subsets of $X$ called blocks such that every 3-element subset is either in a block or a hole but not both. In this article we investigate the existence and non-existence of these designs. We settle all parameter situations on at most 24 points, with 6 exceptions. A uniform group divisible Steiner quadruple system is a system in which all the holes have equal size. These were called by Mills $G$-designs and their existence is completely settled in this article.
\end{abstract}

\section{Introduction}

Given a partition $\mathcal{H}=\left\{H_{1}, H_{2}, \ldots, H_{r}\right\}$ of a set $X$ we say that a subset $T \subseteq X$ is transverse with respect to $\mathcal{H}$ if $\left|T \cap H_{i}\right|=0$ or 1 for each $i=1,2, \ldots, r$.

A group divisible Steiner triple system is a triple $(X, \mathcal{H}, \mathcal{B})$ where $X$ is a $v$-element set of points, $\mathcal{H}=\left\{H_{1}, H_{2}, \ldots, H_{r}\right\}$ is a partition of $X$ into holes and $\mathcal{B}$ is a collection of transverse 3 -element subsets with respect to $\mathcal{H}$ called triples such that:

1. every transverse pair is in a unique triple;

equivalently

2. every pair is either in a hole or a triple, but not both.

Following these two equivalent definitions we see that there are two natural ways to generalize the concept of group divisible Steiner triple systems.

First, a transverse quadruple system is a triple $(X, \mathcal{H}, \mathcal{B})$ where $X$ is a $v$-element set of points, $\mathcal{H}=\left\{H_{1}, H_{2}, \ldots, H_{r}\right\}$ is a partition of $X$ into holes and $\mathcal{B}$ is a collection of transverse 4-element subsets with respect to $\mathcal{H}$ called quadruples such that every transverse 3 -element subset is in exactly one quadruple. 
This article will concentrate on the second generalization. A group divisible Steiner quadruple system, is a triple $(X, \mathcal{H}, \mathcal{B})$ where $X$ is a $v$-element set of points, $\mathcal{H}=$ $\left\{H_{1}, H_{2}, \ldots, H_{r}\right\}$ is a partition of $X$ into holes and $\mathcal{B}$ is a collection of 4 -element subsets of $X$ called blocks such that every 3 -element subset is either in a block or a hole but not both.

Let $h_{i}=\left|H_{i}\right|$ be the size of the hole $H_{i} \in \mathcal{H}$. The type of a quadruple system is the multi-set $\left\{h_{1}, h_{2}, \ldots, h_{r}\right\}$ of hole sizes. It is our custom to write $s_{1}^{u_{1}} s_{2}^{u_{2}} \ldots s_{m}^{u_{m}}=h_{1} h_{2} \cdots h_{r}$ for the type of a quadruple system with $u_{i}$ holes of size $s_{i}, i=1,2, \ldots, m$. If all the holes have the same size $h$, then the quadruple system is said to be uniform. Such a system would have type $h^{u}$ for some $u$.

A transverse Steiner quadruple system of type $s_{1}^{u_{1}} s_{2}^{u_{2}} \ldots s_{m}^{u_{m}}$ is denoted by $\operatorname{TSQS}\left(s_{1}^{u_{1}} s_{2}^{u_{2}} \ldots s_{m}^{u_{m}}\right)$, and a group divisible Steiner quadruple system of the same type is denoted by GSQS $\left(s_{1}^{u_{1}} s_{2}^{u_{2}} \ldots s_{m}^{u_{m}}\right)$. We may also write GSQS $\left(h_{1} h_{2} \cdots h_{r}\right)$ for a GSQS on $r$ holes $h_{1}, h_{2}, \ldots, h_{r}$. Mills was the first to study uniform TSQS, and uniform GSQS he called them $H$-designs and $G$-designs respectively, [21, 22]. (He also calls them $H$ and $G$ systems.) The current status on the existence of $H$-designs is given in Theorems 2,3 , and 4. The existence of $G$-designs is completely settled in Theorem 10 .

Because every block of a GSQS contains exactly 4 triples and these triples are not in any hole, it is easy to count the number of blocks.

Theorem 1 There are exactly

$$
b=|\mathcal{B}|=\frac{1}{4}\left(\left(\begin{array}{l}
v \\
3
\end{array}\right)-\sum_{i=1}^{r}\left(\begin{array}{c}
h_{i} \\
3
\end{array}\right)\right)
$$

blocks in a GSQS $\left(h_{1} h_{2} \cdots h_{r}\right)$ and consequently,

$$
\left(\begin{array}{l}
v \\
3
\end{array}\right) \equiv \sum_{i=1}^{r}\left(\begin{array}{c}
h_{i} \\
3
\end{array}\right) \quad(\bmod 4) .
$$

The following result on transverse Steiner quadruple systems was established by Mills in $[21]$.

Theorem 2 (Mills, 1990) For $u \geq 4, u \neq 5$, a TSQS $\left(h^{u}\right)$ exists if and only if hu is even and $h(u-1)(u-2) \equiv 0 \quad(\bmod 3)$.

As reported in Lauinger et. al., 2005 [20], with reference to the case $u=5$, Mills [21] notes the non-existence of a TSQS of type $2^{5}$ (proved by Stanton and Mullin [24]). The existence of a TSQS of type $6^{5}$ is shown by Mills in Lemma 7 of [22]. Mills reports the existence of a TSQS of type $4^{5}$ but does not present a construction for it. Lauinger, Kreher, Rees, and Stinson give a construction in [20]. (Hartman and Phelps (Section 7 in [12]) comment on the relevance of this design to the Granville-Hartman bound for embeddings of SQSs.) The following result was obtained by Lauinger, Kreher, Rees, and Stinson in $[20]$. 
Theorem 3 (Lauinger, et. al., 2005 [20]) There exists a TSQS of type $h^{5}$ for all $h \equiv$ $0,4,6$, or $8(\bmod 12)$.

This result was recently improved by L. Ji [14].

Theorem 4 (L. Ji, 2008 [14]) There exists a TSQS of type $h^{5}$ if $h$ is even, $h \neq 2$ and $h \not \equiv 10$ or $26 \quad(\bmod 48)$.

Let $(X, \mathcal{H}, \mathcal{B})$ be a GSQS and let $S \subset X$. The derived design with respect to $S$ is a triple $\left(X^{\prime}, \mathcal{H}^{\prime}, \mathcal{B}^{\prime}\right)$ where $X^{\prime}=X \backslash S, \mathcal{B}^{\prime}=\{\mathcal{B} \backslash S: S \subset B\}$, and $\mathcal{H}^{\prime}=\left\{H_{i} \cap X^{\prime}: H_{i} \in \mathcal{H}\right\}$. Following are some necessary conditions for the existence of a GSQS that can be obtained by considering derived designs.

Theorem 5 If a GSQS $\left(h_{1} h_{2} \cdots h_{r}\right)$ exists, then $v=h_{1}+h_{2}+\cdots+h_{r}$ is even.

Proof. Consider the derived design with respect to $S=\{x, y\}$ such that $x \in H_{i}$ and $y \in H_{j}$, for some $i \neq j$. This derived design forms a matching on the other $v-2$ points. Therefore, $v-2 \equiv 0 \quad(\bmod 2)$, so $v$ is even.

Example 6 If all the holes have size 1, then a GSQS is an $\operatorname{SQS}(v)$. Hence a GSQS $\left(1^{v}\right)$ exists if and only if $v \equiv 2$ or $4 \quad(\bmod 6)$.

Lemma 7 If a GSQS $\left(h_{1} h_{2} \cdots h_{r}\right)$ exists, then all of the holes which have size greater than one are even.

Proof. Let $v=h_{1}+h_{2}+\cdots+h_{r}$ be the number of points. Consider the derived design with respect to $S=\{x, y\}$ such that $x, y \in H_{i}$ for some $i$, where $\left|H_{i}\right|>2$ and $x \neq y$. This design forms a matching on the other $v-\left|H_{i}\right|$ points. Therefore, $v-\left|H_{i}\right| \equiv 0 \quad(\bmod 2)$. Thus applying Theorem 5 we see that $\left|H_{i}\right|$ is even.

The next theorem provides necessary conditions for the existence of a GSQS $\left(h_{1} h_{2} \cdots h_{r}\right)$ on $v$ points. Note in particular the condition provided by Equation 1 is redundant, because the number of points and all the holes of size larger than 1 are even.

Theorem 8 (Necessary conditions) If a $\operatorname{GSQS}\left(h_{1} h_{2} \cdots h_{r}\right)$ on $v$ points exists, then

1. either $v \equiv 0 \quad(\bmod 6)$ and all the holes are of size $0 \quad(\bmod 6)$,

2. or $v \equiv 2$ or $4 \quad(\bmod 6)$ and $h_{i}=1$ or $h_{i} \equiv 2$ or $4 \quad(\bmod 6)$, for all $i=1 \ldots r$.

Proof. If $(X, \mathcal{H}, \mathcal{B})$ is a group divisible Steiner quadruple system on $v=|X|$ points and $x \in H \in \mathcal{H}$, then the derived design with respect to $x$ is an incomplete Steiner triple system of order $v-1$ with a hole of size $|H|-1$. The number of triples in such a system is

$$
\frac{1}{3}\left\{\left(\begin{array}{c}
v-1 \\
2
\end{array}\right)-\left(\begin{array}{c}
|H|-1 \\
2
\end{array}\right)\right\} \text {. }
$$


Thus if $|H|=1$ or 2 , we see that $\frac{1}{3}\left(\begin{array}{c}v-1 \\ 2\end{array}\right)$ is an integer and consequently, because $v$ is even by Theorem 5 we have $v \equiv 2$ or $4(\bmod 6)$. If $|H|>2$, then we obtain $|H|^{2} \equiv v^{2}$ $(\bmod 3)$. Consequently, if there is a hole of size $0(\bmod 6)$, then all the holes must have size $0 \quad(\bmod 6)$ and also $v \equiv 0 \quad(\bmod 6)$.

Now suppose $\left|H_{i}\right| \equiv 2$ or $4 \quad(\bmod 6)$ for some $i$ and $\left|H_{i}\right|>2$, then $v^{2} \equiv 4 \quad(\bmod 6)$ and it follows that $v \equiv 2$ or $4(\bmod 6)$.

Remark 9 Kreher and Rees [16] have shown that the maximum size of a block in any Steiner $t$-wise balanced design has size at most $v / 2$ when $t$ is odd. (Kramer [18], had already established this for $t=3$.) Thus if there exists a GSQS on $v$ points, then the maximum size of a hole is $v / 2$.

\section{Existence and non-existence results}

Consider any pair of holes $H_{1}$ and $H_{2}$ that have the same even cardinality $m$. Construct the complete graph $G_{i}$ on hole $H_{i}, i=1,2$. Let $\mathcal{F}_{i}=\left\{F_{i_{1}}, F_{i_{2}}, \ldots, F_{i_{m-1}}\right\}$ be a onefactorization for $i=1$ and $i=2$. Pair the one-factors of $\mathcal{F}_{1}$ and $\mathcal{F}_{2}$ to construct blocks. That is for $i \neq j$ we take as blocks $\{a, b, c, d\}$ where $\{a, b\} \in \mathcal{F}_{i_{k}}$ and $\{c, d\} \in \mathcal{F}_{j_{k}}$, $k=1,2, \ldots, m-1$. These blocks will cover triples consisting of two points from $H_{1}$ and one point from $H_{2}$ or one point from $H_{1}$ and two points from $H_{2}$. We refer to this construction as the doubling one-factorization or DOF construction, and write $\operatorname{DOF}\left(H_{1}, H_{2}\right)$ for the set of quadruples so obtained.

The uniform case, where all the holes are of the same size, can be completely settled by using candelabra quadruple systems, Theorem 2, the doubling one-factorization construction and a result of Hartman or Lenz.

Necessary conditions for the existence of a uniform GSQS $\left(g^{u}\right)$ can be obtained as follows. If $g=1$, then a $\operatorname{GSQS}\left(g^{u}\right)$ is an $\operatorname{SQS}(u)$ which exists if and only if $u=2$ or 4 $(\bmod 6)$. So suppose $g>1$ and a GSQS $\left(g^{u}\right)$ exists. Then $g$ is even by Lemma 7 and we consider the derived design with respect to a point. We obtain a $2-\left(g u-1,\left\{3,(g-1)^{\star}\right\}, 1\right)$ design, i.e. an incomplete Steiner triple system of order $g u-1$ with a hole of size $g-1$. The number of triples in such a design is

$$
\frac{1}{3}\left\{\left(\begin{array}{c}
g u-1 \\
2
\end{array}\right)-\left(\begin{array}{c}
g-1 \\
2
\end{array}\right)\right\} .
$$

Hence

$$
(g u-1)(g u-2) \equiv(g-1)(g-2) \quad(\bmod 3) .
$$

Thus considering the possibilities for $g \quad(\bmod 3)$, we see that

$$
g(u-1)(u-2) \equiv 0 \quad(\bmod 3) .
$$

A Candelabra quadruple system of type $\left(g_{1}^{n_{1}} g_{2}^{n_{2}} \cdots g_{k}^{n_{k}}: s\right)$ denoted by $\operatorname{CQS}\left(g_{1}^{n_{1}} g_{2}^{n_{2}} \cdots g_{k}^{n_{k}}: s\right)$ is a quadruple $(X, S, \mathcal{H}, \mathcal{B})$ where $X$ is a set of size $v=$ 
$s+n_{1} g_{1}+n_{2} g_{2}+\cdots+n_{k} g_{k}, S$ is a subset of $X$ of size $s$, and $\mathcal{H}=\left\{H_{1}, H_{2}, \ldots, H_{r}\right\}$ is a partition of $X \backslash S$ of type $g_{1}^{n_{1}} g_{2}^{n_{2}} \cdots g_{k}^{n_{k}}, r=n_{1}+n_{2}+\cdots+n_{k}$. The set $\mathcal{B}$ contains 4-element subsets of $X$ called blocks, such that every 3 -element subset $T \subseteq X$ with $\left|T \cap\left(S \cup H_{i}\right)\right|<3$ for all $i$ is contained in a unique block and no 3-element subset of $S \cup H_{i}$ is contained in any block. The members of $\mathcal{H}$ are called the branches and $S$ is called the stem.

A short survey of candelabra quadruple systems can be found in [12]. Here we will use the existence of a CQS $\left(g^{4}: s\right)$ for all even $g$ and $s$ with $g \geq s$, which was established by Granville and Hartman [7]. Starting with a CQS $\left(g^{4}: g\right)$, if we apply the DOF construction between each branch and the stem, then the result is a GSQS $\left(g^{5}\right)$. Theorem 2 of Mills establishes necessary and sufficient conditions for the existence of TSQS $\left(g^{u}\right)$ for all $u \geq 4$, and $u \neq 5$. Thus when $g$ is even we can again apply the DOF construction between each pair of holes to obtain a GSQS $\left(g^{u}\right)$. The existence of a $\operatorname{GSQS}\left(g^{3}\right)$ for $g \equiv 0 \quad(\bmod 6)$ has been proved by Hartman [9], also by Lenz [19]. It is easy to see that a GSQS $\left(g^{2}\right)$ exists if and only if $g$ is even, by applying the DOF construction.

The combination of the above results yields the following theorem.

Theorem 10 There exists a GSQS $\left(g^{u}\right)$ if and only if either $g=1$ and $u=2$ or 4 $(\bmod 6)$, or $g$ is even and $g(u-1)(u-2) \equiv 0 \quad(\bmod 3)$.

This theorem surprisingly does not already appear in the literature.

A hole $H$ of a GSQS can be filled in with any GSQS of order $|H|$ thus we obtain the following useful theorem.

Theorem 11 If there exists a GSQS $\left(h_{1} h_{2} \cdots h_{r}\right)$ and a GSQS $\left(g_{1} g_{2} \cdots g_{s}\right)$, where $h_{r}=$ $g_{1}+g_{2}+\cdots+g_{s}$, then there exists a GSQS $\left(h_{1} h_{2} \cdots h_{r-1} g_{1} g_{2} \cdots g_{s}\right)$.

In particular holes of size 2 can be replaced by two holes of size 1 , and in this case the converse is also true, because holes of size 1 and 2 do not contain any triple. Thus we have the rather obvious but useful pair of theorems.

Theorem $12 A$ GSQS $\left(1^{x} 2^{y} g_{1} g_{2} \cdots g_{r}\right)$ exists if and only if a GSQS $\left(1^{x+2} 2^{y-1} g_{1} g_{2} \cdots g_{r}\right)$ exists, $g_{i}>2, i=1,2, \ldots, r$.

Theorem 13 There exists $a \operatorname{GSQS}\left(1^{u} 2^{w}\right)$ if and only if $u+2 w \equiv 2$ or $4(\bmod 6)$, and $a \operatorname{GSQS}\left(1^{u} 2^{w} 4^{1}\right)$ exists if and only if $u+2 w \equiv 0$ or $4 \quad(\bmod 6), u, w \geq 0$.

Proof. A GSQS $\left(1^{u} 2^{w}\right)$ exists if and only if $u+2 w \equiv 2$ or $4 \quad(\bmod 6)$ because it is just an $S Q S(u+2 w)$. If $u+2 w \equiv 0$ or $4 \quad(\bmod 6)$, then $u+2 w+4 \equiv 2$ or $4 \quad(\bmod 6)$, so there exists an SQS $(u+2 w+4)$. Remove one block from this design, and form the hole of size 4 with the points from this block. Form the remaining $u$ holes of size 1 and $w$ holes of size 2 with the remaining points. Every triple is contained in exactly one block, so the only triples that are contained in a hole are the ones that are in the hole of size 4. However, this block has been removed from the design, so what remains is a GSQS $\left(1^{u} 2^{w} 4^{1}\right)$. 
Conversely, given a GSQS $\left(1^{u} 2^{w} 4^{1}\right)$ we can fill in the hole of size 4 with a quadruple and obtain a $\operatorname{SQS}(u+2 w+4)$. Therefore, $u+2 w+4 \equiv 2$ or $4 \quad(\bmod 6)$. Thus, $u+2 w \equiv 0$ or $4(\bmod 6)$.

This result can be generalized to Steiner quadruple systems with $m$ disjoint blocks.

Theorem $14 A \operatorname{SQS}(v)$ with $m$ disjoint blocks, exists if and only if a GSQS $\left(1^{x} 2^{y} 4^{m}\right)$ exists where, $x, y>0$ and $x+2 y+4 z=v$.

Proof. Remove the $m$ disjoint blocks in the SQS $(v)$ to form $m$ holes of size 4 . On the remaining $v-4 m$ points form $y$ holes of size 2 and $x=v-2 y-4 m$ holes of size 1 . Every triple is contained in exactly one block, so the only triples that are contained in holes are the ones that are in the holes of size 4. However, these blocks have been removed from the design, so what remains is a $\operatorname{GSQS}\left(1^{x} 2^{y} 4^{m}\right)$.

Conversely given a GSQS $\left(1^{x} 2^{y} 4^{m}\right)$ where, $x, y>0$ and $x+2 y+4 z=v$, fill in the holes of size 4 with a block to obtain a $\operatorname{SQS}(v)$.

Using Theorem 14 we can show that the necessary conditions given in Theorem 8 are sufficient, when the number of points is at most 18 .

Theorem $15 A$ GSQS $\left(h_{1} h_{2} \cdots h_{r}\right)$ on $v \leq 18$ points exists if and only if

1. either $v \equiv 0 \quad(\bmod 6)$ and all the holes are of size $0 \quad(\bmod 6)$,

2. or $v \equiv 2$ or $4 \quad(\bmod 6)$ and $h_{i}=1$ or $h_{i} \equiv 2$ or $4 \quad(\bmod 6)$, for all $i=1 \ldots r$.

Proof. These conditions were shown to be necessary in Theorem 8. For the converse first assume $v \leq 18$ and $v \equiv 0 \quad(\bmod 6)$. Then $v \in\{6,12,18\}$ and we see by Remark 9 , that all the holes have size 6. Hence the GSQS is uniform and thus exists by Theorem 10. If $v \in\{1,2,4\}$, the required GSQS trivially exists. If $v \in\{8,10,14\}$ the maximum size of a hole is 4 . Thus it is sufficient to construct a $\operatorname{SQS}(v)$ with $m=\left\lfloor\frac{v}{4}\right\rfloor$ and use Theorem 14 .

The unique SQS(8) and SQS(10) can both easily be shown to have 2 disjoint blocks.

An $\operatorname{SQS}(14)$ on $V=\left\{x_{i}: x \in \mathbb{Z}_{7}, i \in \mathbb{Z}_{2}\right\}$ is obtained when the five base blocks

$$
\left\{3_{0}, 4_{0}, 3_{1}, 4_{1}\right\} \quad\left\{0_{0}, 1_{0}, 2_{0}, 4_{0}\right\} \quad\left\{0_{0}, 1_{1}, 2_{1}, 4_{1}\right\} \quad\left\{4_{0}, 5_{0}, 2_{1}, 3_{1}\right\} \quad\left\{5_{0}, 6_{0}, 1_{1}, 2_{1}\right\}
$$

are developed with the automorphisms $x_{i} \mapsto(3 x)_{i}$ and $x_{i} \mapsto(x+1)_{i}$. This SQS(14) contains the disjoint quadruples

$$
\left\{3_{0}, 4_{0}, 3_{1}, 4_{1}\right\} \quad\left\{2_{0}, 5_{0}, 2_{1}, 5_{1}\right\} \quad\left\{1_{0}, 6_{0}, 1_{1}, 6_{1}\right\},
$$

which can be seen by developing the first base block with the first automorphism. This SQS(14) appears in Example 5.29 of Part II of the CRC Handbook of Combinatorial Designs [5].

If $v=16$ and the maximum size of a hole is 4 , we observe that a SQS(16) with 4 disjoint blocks is equivalent to a $\operatorname{GSQS}\left(4^{4}\right)$, which exists by Theorem 10 . The desired GSQS on 16 points can then be constructed by using Theorem 14. If a hole of size 8 is required, we first construct a GSQS $\left(8^{2}\right)$ using Theorem 10. A hole of size 8 can then be filled in with a GSQS on 8 points to obtain the desired GSQS on 16 points.

The necessary conditions provided by Theorem 8 are in general not sufficient as the following theorem shows. 


\section{Theorem 16}

a. If a GSQS has only three holes, then it has type $h g^{2}, g \leq 2 h \leq 4 g$, and $h g(h+g) \equiv 0$ $(\bmod 3)$.

b. There does not exist a GSQS of type $1^{x} 2^{y} 4^{z} b c$, when $c>b>4=x+2 y+4 z$.

Proof. For Part a, let $(X, \mathcal{B}, \mathcal{H})$ be a GSQS $(a b c)$ with holes $A, B, C$, where $a=|A|$, $b=|B|$ and $c=|C|$. We classify triples and possible quadruples according to how they intersect $A, B, C$. For $m=3$, 4, let

$$
\mathcal{S}_{m}(i, j, k)=\{S \subseteq X:|S|=3,(|S \cap A|,|S \cap B|,|S \cap C|)=(i, j, k), i+j+k=m\}
$$

The cardinality of $\mathcal{S}_{m}(i, j, k)$ is $\left(\begin{array}{l}a \\ i\end{array}\right)\left(\begin{array}{l}b \\ j\end{array}\right)\left(\begin{array}{l}c \\ k\end{array}\right)$. Let $M$ be the incidence matrix defined by

$$
M\left[S_{3}(i, j, k), S_{4}\left(i^{\prime}, j^{\prime}, k^{\prime}\right)\right]=\left|\left\{T \in S_{3}(i, j, k): T \in Q\right\}\right|,
$$

where $Q \in \mathcal{S}_{4}\left(i^{\prime}, j^{\prime}, k^{\prime}\right)$ is any representative. Then $M$ is the 7 by 6 matrix:

\begin{tabular}{c|cccccc} 
& $\mathcal{S}_{4}(2,2,0)$ & $\mathcal{S}_{4}(2,0,2)$ & $\mathcal{S}_{4}(0,2,2)$ & $\mathcal{S}_{4}(2,1,1)$ & $\mathcal{S}_{4}(1,2,1)$ & $\mathcal{S}_{4}(1,1,2)$ \\
\hline $\mathcal{S}_{3}(2,1,0)$ & 2 & 0 & 0 & 1 & 0 & 0 \\
$\mathcal{S}_{3}(2,0,1)$ & 0 & 2 & 0 & 1 & 0 & 0 \\
$\mathcal{S}_{3}(0,2,1)$ & 0 & 0 & 2 & 0 & 1 & 0 \\
$\mathcal{S}_{3}(1,2,0)$ & 2 & 0 & 0 & 0 & 1 & 0 \\
$\mathcal{S}_{3}(1,0,2)$ & 0 & 2 & 0 & 0 & 0 & 1 \\
$\mathcal{S}_{3}(0,1,2)$ & 0 & 0 & 2 & 0 & 0 & 1 \\
$\mathcal{S}_{3}(1,1,1)$ & 0 & 0 & 0 & 2 & 2 & 2
\end{tabular}

Let

$$
\begin{aligned}
W= & {\left[\left|\mathcal{S}_{3}(2,1,0)\right|,\left|\mathcal{S}_{3}(2,0,1)\right|,\left|\mathcal{S}_{3}(0,2,1)\right|,\left|\mathcal{S}_{3}(1,2,0)\right|\right.} \\
& \left.\left|\mathcal{S}_{3}(1,0,2)\right|,\left|\mathcal{S}_{3}(0,1,2)\right|,\left|\mathcal{S}_{3}(1,1,1)\right|\right] \\
= & {\left[\left(\begin{array}{c}
a \\
2
\end{array}\right) b,\left(\begin{array}{c}
a \\
2
\end{array}\right) c,\left(\begin{array}{c}
b \\
2
\end{array}\right) c, a\left(\begin{array}{c}
b \\
2
\end{array}\right), a\left(\begin{array}{c}
c \\
2
\end{array}\right), b\left(\begin{array}{c}
c \\
2
\end{array}\right), a b c\right] }
\end{aligned}
$$

and let

$$
U=\left[u_{\mathcal{S}_{4}(2,2,0)}, u_{\mathcal{S}_{4}(2,0,2)}, u_{\mathcal{S}_{4}(0,2,2)}, u_{\mathcal{S}_{4}(2,1,1)}, u_{\mathcal{S}_{4}(1,2,1)}, u_{\mathcal{S}_{4}(1,1,2)}\right],
$$

where $u_{\mathcal{S}_{4}(i, j, k)}=\left|\mathcal{B} \cap \mathcal{S}_{4}(i, j, k)\right|$. Then

$$
M U=W
$$

Observe that $E=[1,-1,1,-1,1,-1,0]^{T}$ is in the null-space of $M^{T}$. Thus

$\overrightarrow{0}=E^{T} M U=E^{T} W=\left(\begin{array}{l}a \\ 2\end{array}\right) b-\left(\begin{array}{l}a \\ 2\end{array}\right) c+\left(\begin{array}{l}b \\ 2\end{array}\right) c-a\left(\begin{array}{l}b \\ 2\end{array}\right)+a\left(\begin{array}{l}c \\ 2\end{array}\right)-b\left(\begin{array}{l}c \\ 2\end{array}\right)=\frac{1}{2}(a-b)(a-c)(b-c)$. 
Thus at least two of the hole sizes must be equal. We will assume $c=b$. The columns of $M$ are easily seen to be linearly independent. Hence there is a unique solution to the equation $M U=W$. When $c=b$ this solution is

$$
\begin{gathered}
U=\left[\frac{a b(a+b-3)}{12}, \frac{a b(a+b-3)}{12}, \frac{b\left(a^{2}-3 b-2 a b+3 b^{2}\right)}{12},\right. \\
\left.\frac{a(2 a-b) b}{6}, \frac{a(2 b-a) b}{6}, \frac{a(2 b-a) b}{6}\right] .
\end{gathered}
$$

The entries of $U$ are non-negative integers. So it follows that $b \leq 2 a \leq 4 b$ and that $a b(a+b) \equiv 0 \quad(\bmod 3)$. (Recall Theorem 8 says either both $a$ and $b$ are $0 \quad(\bmod 3)$ or neither are.)

For Part b we repeat nearly the same argument as was given in Part a. Let $(X, \mathcal{B}, \mathcal{H})$ be a GSQS of order $4+b+c$, with holes $B$ and $C$, where $b=|B|$ and $c=|C|$ and we let $A$ be the remaining 4 points. Note that the set $A$ cannot be a quadruple for then there would exist a SQS of type $4^{1} a^{1} b^{1}$, which is impossible by Part a. We again classify triples and possible quadruples according to how they intersect $A, B, C$, and construct the incidence matrix $M$ in the same manner as in Part a. Then $M$ is the 8 by 8 matrix:

\begin{tabular}{c|cccccccc} 
& $\mathcal{S}_{4}(2,2,0)$ & $\mathcal{S}_{4}(2,0,2)$ & $\mathcal{S}_{4}(0,2,2)$ & $\mathcal{S}_{4}(2,1,1)$ & $\mathcal{S}_{4}(1,2,1)$ & $\mathcal{S}_{4}(1,1,2)$ & $\mathcal{S}_{4}(3,1,0)$ & $\mathcal{S}_{4}(3,0,1)$ \\
\hline $\mathcal{S}_{3}(2,1,0)$ & 2 & 0 & 0 & 1 & 0 & 0 & 3 & 0 \\
$\mathcal{S}_{3}(2,0,1)$ & 0 & 2 & 0 & 1 & 0 & 0 & 0 & 3 \\
$\mathcal{S}_{3}(0,2,1)$ & 0 & 0 & 2 & 0 & 1 & 0 & 0 & 0 \\
$\mathcal{S}_{3}(1,2,0)$ & 2 & 0 & 0 & 0 & 1 & 0 & 0 & 0 \\
$\mathcal{S}_{3}(1,0,2)$ & 0 & 2 & 0 & 0 & 0 & 1 & 0 & 0 \\
$\mathcal{S}_{3}(0,1,2)$ & 0 & 0 & 2 & 0 & 0 & 1 & 0 & 0 \\
$\mathcal{S}_{3}(1,1,1)$ & 0 & 0 & 0 & 2 & 2 & 2 & 0 & 0 \\
$\mathcal{S}_{3}(3,0,0)$ & 0 & 0 & 0 & 0 & 0 & 0 & 1 & 1
\end{tabular}

Let

$$
\begin{aligned}
W & =\left[\left|\mathcal{S}_{3}(2,1,0)\right|,\left|\mathcal{S}_{3}(2,0,1)\right|,\left|\mathcal{S}_{3}(0,2,1)\right|,\left|\mathcal{S}_{3}(1,2,0)\right|\right. \\
& \left.\left|\mathcal{S}_{3}(1,0,2)\right|,\left|\mathcal{S}_{3}(0,1,2)\right|,\left|\mathcal{S}_{3}(1,1,1)\right|,\left|\mathcal{S}_{3}(3,0,0)\right|\right] \\
= & {\left[6 b, 6 c,\left(\begin{array}{c}
b \\
2
\end{array}\right) c, 4\left(\begin{array}{c}
b \\
2
\end{array}\right), 4\left(\begin{array}{c}
c \\
2
\end{array}\right), b\left(\begin{array}{c}
c \\
2
\end{array}\right), 4 b c, 4\right] }
\end{aligned}
$$

and let

$$
U=\left[u_{\mathcal{S}_{4}(2,2,0)}, u_{\mathcal{S}_{4}(2,0,2)}, u_{\mathcal{S}_{4}(0,2,2)}, u_{\mathcal{S}_{4}(2,1,1)}, u_{\mathcal{S}_{4}(1,2,1)}, u_{\mathcal{S}_{4}(1,1,2)}, u_{\mathcal{S}_{4}(3,1,0)}, u_{\mathcal{S}_{4}(3,0,1)}\right]
$$

where $u_{\mathcal{S}_{4}(i, j, k)}=\left|\mathcal{B} \cap \mathcal{S}_{4}(i, j, k)\right|$. Then

$$
M U=W
$$

It is easy to see that $M$ is nonsigular and thus has a unique solution $U$. Considering $E=[1,-1,1,-1,1,-1,0,3]^{T}$, we see that

$$
6 u_{\mathcal{S}_{4}(3,1,0)}=[0,0,0,0,0,0,6,0] U=E^{T} M U=E^{T} W=\frac{1}{2}(4-b)(4-c)(b-c)+12 .
$$


Because $4<b<c$, the quantities $4-b, 4-c$ and $b-c$ are all negative.

If $v \equiv 2,4 \quad(\bmod 6)$, then $b, c \equiv 2,4 \quad(\bmod 6)$, by Theorem 8 . Thus $4-b, 4-c \leq-4$ and $b-c \leq-2$. Hence

$$
6 u_{\mathcal{S}_{4}(3,1,0)}=\frac{1}{2}(4-b)(4-c)(b-c)+12 \leq \frac{1}{2}(-4)(-4)(-2)+12=-4
$$

Thus no such GSQS can exist.

If $v \equiv 0 \quad(\bmod 6)$, then $b, c \equiv 0 \quad(\bmod 6)$, by Theorem 8 . Considering $E^{\prime}=$ $[1,-1,1,-1,1,-1,-3,0]^{T}$, we see that

$$
-6 u_{\mathcal{S}_{4}(3,0,1)}=[0,0,0,0,0,0,0,-6] U=E^{\prime T} M U=E^{\prime T} W=\frac{1}{2}(4-b)(4-c)(b-c)-12 .
$$

SO

$$
(4-b)(4-c)(c-b)=12 u_{\mathcal{S}_{4}(3,0,1)}-24 \equiv 0 \quad(\bmod 12) .
$$

Thus $b \equiv c \quad(\bmod 12)$ and consequently $b-c \leq-12$. Also we know $4-b, 4-c \leq-2$. Hence

$$
6 u_{\mathcal{S}_{4}(3,1,0)}=\frac{1}{2}(4-b)(4-c)(b-c)+12 \leq \frac{1}{2}(-2)(-2)(-12)+12=-12
$$

Thus no such GSQS can exist.

Corollary 17 GSQS $\left(1^{2} g^{2}\right)$ and a GSQS $\left(2^{1} g^{2}\right)$ exist, if and only if $g=0,1$ or 4 .

Proof. If $g=0$, then observe that both a $\operatorname{GSQS}\left(1^{2}\right)$ and $\operatorname{GSQS}\left(2^{1}\right)$ trivialy exist. So suppose $g \neq 0$ and note that a GSQS $\left(1^{2} g^{2}\right)$ exists if and only if a GSQS $\left(2^{1} g^{2}\right)$ exists. To the latter which we may apply Theorem 16 a and thus $g=1$ or 4 . A GSQS $\left(2^{1} 4^{2}\right)$ and a GSQS $\left(1^{2} 4^{2}\right)$ are constructed in Theorem 15. A single quadruple of size 4 is a $\operatorname{GSQS}\left(2^{1} 1^{2}\right)$ and a GSQS $\left(1^{4}\right)$.

Theorem $18 A$ GSQS $\left(1^{u} 2^{w} g^{1}\right)$ exists if and only if there exists a $\mathrm{SQS}(u+2 w+g)$ containing a sub-SQS $(g)$.

Proof. If a GSQS $\left(1^{u} 2^{w} g^{1}\right)$ exists, then $g \equiv 2,4 \quad(\bmod 6)$, by Theorem 8 . Hence there exists a SQS $(g)$. We now fill in the hole of size $g$ with a $\operatorname{SQS}(g)$. This results in a $\operatorname{SQS}(u+2 w+g)$, because holes of size 1 and 2 contain no triples.

Conversely if a $\operatorname{SQS}(u+2 w+g)$ exists and contains a $\operatorname{SQS}(g)$ as a subsystem, then removing the quadruples in the $\operatorname{SQS}(g)$ produces a GSQS $\left(1^{u+2 w} g^{1}\right)$ which is also a GSQS $\left(1^{u} 2^{w} g^{1}\right)$, because holes of size 1 or 2 do not contain any triples.

Hartman [10] and Lenz [19] have shown that whenever $v \geq 16$, then there is a SQS $(v)$ containing a sub-GSQS(8). The sub-design problem was thoroughly investigated by Granville and Hartman [7]. In particular the existence of a SQS(22) containing a subSQS(10) can be deduced from their results. 
A $\operatorname{SQS}(v)$ has $b_{i}=\left(\begin{array}{c}v-i \\ 3-i\end{array}\right) /\left(\begin{array}{c}4-i \\ 3-i\end{array}\right)$ quadruples containing a given set of $i$ points, for $i=0,1,2,3$. If it contains a sub-SQS $(w)$, then an easy inclusion-exclusion argument shows that it has

$$
b_{0}-w b_{1}+\left(\begin{array}{l}
w \\
2
\end{array}\right) b_{2}-\left(\begin{array}{l}
w \\
3
\end{array}\right)+\frac{1}{4}\left(\begin{array}{l}
w \\
3
\end{array}\right)
$$

quadruples disjoint from the sub-system. This quantity can easily be shown to be positive. Consequently we have the following result by removing a quadruple disjoint from a subsystem.

Theorem 19 If there exists a SQS $(v)$ containing a sub-SQS $(g)$, then there exists a GSQS $\left(1^{x} 2^{y} 4^{1} g^{1}\right)$ for all $x, y \geq 0, x+2 y+4+g=v$.

Theorem 20 If there exists a GSQS $\left(h_{1} h_{2} \cdots h_{r}\right)$ and a GSQS $\left(g_{1} g_{2} \cdots g_{s}\right)$, where $v=h_{1}+h_{2}+\cdots+h_{r}=g_{1}+g_{2}+\cdots+g_{s}$, then there exists a GSQS $\left(h_{1} h_{2} \cdots h_{r} g_{1} g_{2} \cdots g_{s}\right)$.

Proof. Because there exists a $\operatorname{GSQS}\left(h_{1} h_{2} \cdots h_{r}\right)(X, \mathcal{H}, \mathcal{B})$ and a $\operatorname{GSQS}\left(g_{1} g_{2} \cdots g_{s}\right)$, $\left(X^{\prime}, \mathcal{H}^{\prime}, \mathcal{B}^{\prime}\right)$, the only other triples we need to cover are the ones which have 2 points in $X$ and 1 point in $X^{\prime}$ or 1 point in $X$ and 2 points in $X^{\prime}$. We simply apply the DOF construction between the point set $X$ and the point set $X^{\prime}$. That is we include $\operatorname{DOF}\left(X, X^{\prime}\right)$ as additional quadruples.

Theorem 21 There exists a GSQS $\left(h^{u}(2 h)^{k}\right)$ if and only if $h=1$ and $u+2 k \equiv 2$ or $4(\bmod 6)$ or $h>1$ and $h(u+2 k-1)(u+2 k-2) \equiv 0 \quad(\bmod 3)$ except possibly when $h \equiv 10$ or $26 \quad(\bmod 48)$ and $(u, k) \in\{(3,1),(1,2)\}$.

Proof. When $h=2$ and $u+2 k=5$, the required GSQS is obtained from either Theorem 10 or Theorem 15 . When $h \equiv 10$ or $26(\bmod 48)$, the GSQS is obtained by Theorem 10. Otherwise from Theorems 2 and 4 we see that these conditions imply the existence of a TSQS $\left(h^{u+2 k}\right)$ with holes $H_{i}, G_{j}, G_{j}^{\prime}, i=1,2, \ldots, u, j=1,2, \ldots, k$. We include the additional quadruples in $\operatorname{DOF}\left(H_{i_{1}}, H_{i_{2}}\right), i_{1} \neq i_{2}, \operatorname{DOF}\left(H_{i}, G_{j}\right), \operatorname{DOF}\left(H_{i}, G_{j}^{\prime}\right)$, $\operatorname{DOF}\left(G_{j_{1}}, G_{j_{2}}^{\prime}\right), j_{1} \neq j_{2}$.

If a $\operatorname{GSQS}\left(h^{u}(2 h)^{k}\right)$ exists and $h=1$, then $u+2 k \equiv 2$ or $4 \quad(\bmod 6)$, because it is a $\operatorname{SQS}(u+2 k)$.

If $h>1$, then $h$ is even by Lemma 7 , and we consider the derived design with respect to a point in the hole of size $h$. This forms a $2-\left(h u+2 h k-1,\left\{3,(h-1)^{*}\right\}, 1\right)$ design. It has

$$
\frac{1}{3}\left(\left(\begin{array}{c}
h u+2 h k-1 \\
2
\end{array}\right)-\left(\begin{array}{c}
h-1 \\
2
\end{array}\right)\right)
$$

triples. Therefore $(h(u+2 k)-1)(h(u+2 k)-2) \equiv(h-1)(h-1) \quad(\bmod 3)$. So considering the possibilities for $h \bmod 3$, we see that

$$
h(u+2 k-1)(u+2 k-2) \equiv 0 \quad(\bmod 3) .
$$


Theorem 22 A GSQS of order 20 exists if and only if it has type $1^{a} 2^{b} 4^{c} 8^{d} 10^{e}$, where $a+2 b+4 c+8 d+10 e=20$, and $(d, e) \neq(1,1)$.

Proof. We see from Theorem 10 that a GSQS $\left(4^{5}\right)$ and a GSQS $\left(10^{2}\right)$ exist. A GSQS $\left(4^{1} 8^{2}\right)$ can be constructed using Theorem 21 . Using Theorem 11 we can can construct all possible configurations of holes for GSQS of order 20, except types $1^{2} 8^{1} 10^{1}$ and $2^{1} 8^{1} 10^{1}$ which do not exist by Theroem 16a and Theorem 12 .

Theorem 23 If there exists a $\mathrm{CQS}\left(g^{3}: s\right)$ and an $\operatorname{SQS}(g+s)$ with a sub-SQS $(s)$, then there exists a $\mathrm{SQS}(3 g+s)$ with a sub-SQS $(g+s)$ and 3 disjoint blocks that are disjoint from the subsystem, and therefore $\left.a \operatorname{GSQS}\left(1^{a} 2^{b}\right) 4^{3}(g+s)^{1}\right)$, exists, for all $a+2 b=2 g-s-12$, $a, b \geq 0$.

Proof. If $s=0$, then a $\operatorname{CQS}\left(g^{3}: s\right)$ is a $\operatorname{GSQS}\left(g^{3}\right)$ and we simply fill in the holes with a $\operatorname{SQS}(g)$ that has two disjoint blocks. Now assume $s>0$. There does not exist an SQS(4) with a subsystem, therefore, we must have that $g+s \geq 8$. So if $s=1$, then $g \geq 7$, and if $s \geq 2$, then $g \geq 6$, i.e. $g$ is always at least 6 . Let $H_{1}, H_{2}, H_{3}$ be the three branches of the CQS. Here we denote the type of a set $S$, by $\left(\left|S \cap H_{1}\right|,\left|S \cap H_{2}\right|,\left|S \cap H_{3}\right|\right)$. Let $x=$ the number of quadruples of type $(2,2,0),(2,0,2)$, or $(0,2,2)$, let $y=$ the number of quadruples of type $(2,1,1),(1,2,1)$, or $(1,1,2)$, and let $z=$ the number of quadruples of type $(1,1,1)$. Also let $T_{1}$ be the set of all triples of type $(2,1,0),(2,0,1),(0,2,1),(1,2,0)$, $(0,1,2)$, or $(1,0,2)$, and let $T_{2}$ be the triples of type $(1,1,1)$. Then $\left|T_{1}\right|=6\left(\begin{array}{l}g \\ 2\end{array}\right) g=3 g^{3}-g$, and $\left|T_{2}\right|=g^{3}$. Counting the number of quadruples of each type that cover the triples in $T_{i}$, we obtain the following two equations respectively for $i=1$ and $i=2$ :

$$
\begin{aligned}
4 x+2 y & =3 g^{3}-g . \\
2 y+z & =g^{3}
\end{aligned}
$$

Subtracting yields $4 x=2 g^{3}-g+z$. Therefore, since $g \neq 0$, we have that $x>0$. Consequently we may assume that there is a quadruple $X$ of type $(0,2,2)$. Construct a $\operatorname{SQS}(g+s)$ on $H_{2} \cup S$ that has a sub-SQS $(s)$ on $S$. (If $s=1$, or 2 , the sub-SQS $(s)$ is trivial.) The argument preceding Theorem 19 shows that the $\mathrm{SQS}(g+s)$ contains at least one block, $B_{2}$, disjoint from the sub-system on $S$; and because $g \geq 6$, we may assume that it is also disjoint from $X$. Remove the sub-SQS $(s)$. Do the same thing for $H_{3} \cup S$, obtaining block $B_{3}$. We now have three disjoint blocks: $B_{2}, B_{3}, X$. Now construct a $\operatorname{SQS}(g+s)$ on $H_{1} \cup S$. This yields an $\operatorname{SQS}(3 g+s)$ with a sub-SQS $(g+s)$ and 3 disjoint blocks that are disjoint from the sub-SQS $(g+s)$.

Finally, to construct a GSQS $\left(1^{2 g-s-12} 4^{3}(g+s)^{1}\right)$, we do the following. Remove the sub-SQS $(g+s)$ and the 3 disjoint blocks, and partition the remaining points into $a$ holes of size 1 and $b$ holes of size 2 , where $a+2 b=2 g-s-12, a, b \geq 0$.

Hartman ([11], [9]) gave direct constructions for $\mathrm{CQS}\left(g^{3}: s\right)$ for all even $s$ and all $g \equiv 0$ or $s \quad(\bmod 6)$ with $g \geq s$.

Example 24 Any block of a SQS(10) can be considered as a sub-SQS(4). Thus using the $\mathrm{CQS}\left(6^{3}: 4\right)$ constructed by Hartman and applying Theorem 23 we obtain a $\operatorname{GSQS}\left(4^{3} 10^{1}\right)$. 
The following theorem was proved by Mohacsy and Ray-Chaudhuri [13].

Theorem 25 Let $q$ be a prime power and $v$ be a positive integer. If there is an $S(3, q+$ $1, v+1)$, then there exist

a. a 3-CS of branch type $\left(q^{v}: 1\right)$ with block size $q+1$ and

b. a 3-CS of branch type $\left(v^{q}: 1\right)$ with block size $q+1$.

Example 26 An SQS(8) can easily be shown to have 2 disjoint blocks, and we can see from Theorem 25 that there exists a $\operatorname{CQS}\left(7^{3}: 1\right)$, therefore we can apply Theorem 23 to obtain a GSQS $\left(2^{1} 4^{3} 8^{1}\right)$.

The situation on 22 points is not completely resolved. Using Theorem 21 and the above examples we can construct a $\operatorname{GSQS}\left(2^{1} 4^{5}\right)$, a $\operatorname{GSQS}\left(2^{1} 4^{3} 8^{1}\right)$ and a $\operatorname{GSQS}\left(4^{3} 10^{1}\right)$. Then using Theorem 11 we can can construct all possible configurations of holes for GSQS of order 22 , except when there are 2 holes of size 8 or when there are 2 holes of size 10 . Theorem 17 establishes the non-existence of an order 22 GSQS with two holes of size 10. Thus the only unresolved types of GSQS of order 22 are: $1^{6} 8^{2}, 1^{4} 2^{1} 8^{2}, 1^{2} 2^{2} 8^{2}, 2^{3} 8^{2}$, $1^{2} 4^{1} 8^{2}$ and $2^{1} 4^{1} 8^{2}$.

Following the technique used in Theorem 16 we can suppose the existence of GSQS of order 22 with two holes $B$ and $C$ each of size 8 . Let $A$ be the remaining 6 points and note that the set $A$ cannot contain more than 3 quadruples for if it did a triple would be covered twice. We again classify triples and possible quadruples according to how they intersect $A, B, C$, and construct the incidence matrix $M$ in the same manner as in Theorem 16. Then $M$ is the 8 by 9 matrix:

\begin{tabular}{c|ccccccccc} 
& $\mathcal{S}_{4}(2,2,0) \mathcal{S}_{4}(2,0,2) \mathcal{S}_{4}(0,2,2) \mathcal{S}_{4}(2,1,1)$ & $\mathcal{S}_{4}(1,2,1)$ & $\mathcal{S}_{4}(1,1,2)$ & $\mathcal{S}_{4}(3,1,0)$ & $\mathcal{S}_{4}(3,0,1) \mathcal{S}_{4}(4,0,0)$ \\
\hline $\mathcal{S}_{3}(2,1,0)$ & 2 & 0 & 0 & 1 & 0 & 0 & 3 & 0 & 0 \\
$\mathcal{S}_{3}(2,0,1)$ & 0 & 2 & 0 & 1 & 0 & 0 & 0 & 3 & 0 \\
$\mathcal{S}_{3}(0,2,1)$ & 0 & 0 & 2 & 0 & 1 & 0 & 0 & 0 & 0 \\
$\mathcal{S}_{3}(1,2,0)$ & 2 & 0 & 0 & 0 & 1 & 0 & 0 & 0 & 0 \\
$\mathcal{S}_{3}(1,0,2)$ & 0 & 2 & 0 & 0 & 0 & 1 & 0 & 0 & 0 \\
$\mathcal{S}_{3}(0,1,2)$ & 0 & 0 & 2 & 0 & 0 & 1 & 0 & 0 & 0 \\
$\mathcal{S}_{3}(1,1,1)$ & 0 & 0 & 0 & 2 & 2 & 2 & 0 & 0 & 0 \\
$\mathcal{S}_{3}(3,0,0)$ & 0 & 0 & 0 & 0 & 0 & 0 & 1 & 1 & 4
\end{tabular}

Let

$$
\begin{aligned}
W= & {\left[\left|\mathcal{S}_{3}(2,1,0)\right|,\left|\mathcal{S}_{3}(2,0,1)\right|,\left|\mathcal{S}_{3}(0,2,1)\right|,\left|\mathcal{S}_{3}(1,2,0)\right|,\left|\mathcal{S}_{3}(1,0,2)\right|\right.} \\
& \left.\left|\mathcal{S}_{3}(0,1,2)\right|,\left|\mathcal{S}_{3}(1,1,1)\right|,\left|\mathcal{S}_{3}(3,0,0)\right|,\left|\mathcal{S}_{3}(4,0,0)\right|\right] \\
= & {[120,120,224,168,168,224,384,20] }
\end{aligned}
$$

and let

$$
U=\left[u_{\mathcal{S}_{4}(2,2,0)}, u_{\mathcal{S}_{4}(2,0,2)}, u_{\mathcal{S}_{4}(0,2,2)}, u_{\mathcal{S}_{4}(2,1,1)}, u_{\mathcal{S}_{4}(1,2,1)}, u_{\mathcal{S}_{4}(1,1,2)}, u_{\mathcal{S}_{4}(3,1,0)}, u_{\mathcal{S}_{4}(3,0,1)}, u_{\mathcal{S}_{4}(4,0,0)}\right],
$$

where $u_{\mathcal{S}_{4}(i, j, k)}=\left|\mathcal{B} \cap \mathcal{S}_{4}(i, j, k)\right|$. Then

$$
M U=W .
$$


There are exactly 4 possible solutions to $M U=W$, that have $0 \leq u_{\mathcal{S}_{4}(4,0,0)} \leq 3$. They are:

\begin{tabular}{c|cccccccc}
$u_{\mathcal{S}_{4}(2,2,0)}$ & $u_{\mathcal{S}_{4}(2,0,2)}$ & $u_{\mathcal{S}_{4}(0,2,2)}$ & $u_{\mathcal{S}_{4}(2,1,1)}$ & $u_{\mathcal{S}_{4}(1,2,1)}$ & $u_{\mathcal{S}_{4}(1,1,2)}$ & $u_{\mathcal{S}_{4}(3,1,0)}$ & $u_{\mathcal{S}_{4}(3,0,1)}$ & $u_{\mathcal{S}_{4}(4,0,0)}$ \\
\hline 39 & 39 & 67 & 12 & 90 & 90 & 10 & 10 & 0 \\
40 & 40 & 68 & 16 & 88 & 88 & 8 & 8 & 1 \\
41 & 41 & 69 & 20 & 86 & 86 & 6 & 6 & 2 \\
42 & 42 & 70 & 24 & 84 & 84 & 4 & 4 & 3
\end{tabular}

Several different automorsphism groups were assumed to aide in the computer search for a GSQS with one of the above possible distribution of quadruples. So far all attemps have failed and the construction of an SQS(22) with two disjoint substsyems of size 8 remains an open problem.

The situation for 24 points is easy, because GSQS $\left(12^{2}\right)$ and GSQS $\left(6^{2}\right)$ exist by Theorem 10 .

\section{Final remarks}

The results presented in this article establish necessary and sufficient conditions for the existence of uniform group divisible Steiner quadruple systems, the so called $G$-designs of Mills. Also all parameter situations of arbitrary GSQS on less than 24 points are settled except for 6 open cases on 22 points. The difficulty appears to be the construction of Steiner quadruple systems on $v \equiv 2(\bmod 6)$ points having large subsystems, in particular, two disjoint subsystems of order $(v-6) / 2$.

\section{Acknowledgments}

The authors enjoyed useful discussions with L. Ji and M.S. Gockenbach. The homework assigned by the referee was particularly helpful and we thank him for the inspiration to improve this article.

\section{References}

[1] R.D. Baker, Partitioning the planes of $A G_{2 m}(2)$ into 2-designs, Discrete Math. 15 (1976) 205-211.

[2] K.A. Bush, A generalization of the theorem due to MacNeish, Ann. Math. Stat. 23 (1952), 293-295.

[3] K.A. Bush, Orthogonal arrays of index unity, Ann. Math. Stat. 23 (1952), 426-434.

[4] C.J. Colbourn, Small group divisible designs with block size three. J. Combin. Math. and Combin. Comput. 14 (1993), 153-171.

[5] The CRC handbook of combinatorial designs C.J. Colbourn and J.H. Dinitz (Editors) CRC Press, Boca Raton, 2007. 
[6] C.J. Colbourn, D. G. Hoffman, R. Rees, A new class of group divisible designs with block size three, J. Combin. Theory A 59 (1992), 73-89.

[7] A. Granville and A. Hartman, Subdesigns in Steiner Quadruple Systems, J. Combin. Theory A 56 (1991), 239-270.

[8] H. Hanani, Balanced incomplete block designs and related designs, Discrete Math., 11 (1975), 255-369.

[9] A. Hartman, A general recursive construction for quadruple systems, J. Combin. Theory A, 33 (1982), 121-134.

[10] A. Hartman, Quadruple Systems containing $A G(3,2)$. Discrete math., 39 (1982), 293-299.

[11] A. Hartman, Tripling quadruple systems, Ars Combin., 10 (1980), 225-309.

[12] A. Hartman and K.T. Phelps, Steiner Quadruple Systems, in Contemporary Design Theory: A collection of surveys, Eds. J.H. Dinitz and D.R. Stinson (Wiley 1992), 205-240.

[13] H. Mohacsy and D.K. Ray-Chaudhuri, Candelabra systems and designs, J. Statist. Plann. Inference, 106 (2002), 419-448.

[14] L. Ji, An improvement on H design, J. Combin. designs, to appear.

[15] L. Ji, L. Zhu, Constructions for Steiner quadruple systems with a spanning block design, Discrete Math. 261 (2003), 347-360.

[16] D. Kreher, R. Rees A Hole-Size Bound for Incomplete $t$-Wise Balanced Designs. $J$ Combin Designs 9 (2001), 269-284.

[17] Donald L. Kreher and Douglas R. Stinson, Combinatorial Algorithms: Generation, Enumeration and Search, 1998, CRC Press LLC.

[18] E.S. Kramer, Some results on $t$-wise balanced designs, Ars Combin. 15 (1983) 179192.

[19] H. Lenz, Tripling Steiner Quadruple Systems, Ars. Combin. 20 (1985), 193-202.

[20] K. Lauinger, D. Kreher, R. Rees, D. Stinson, Computing transverse t-designs. J. Combin. Math. and Combin. Comput. 54 (2005), 33-56.

[21] W. H. Mills, On the existence of H-designs, Congressus Numeratium. 79 (1990) 129141.

[22] W. H. Mills, On the covering of triples by quadruples. Congressus Numeratium. 10 (1974) 563-581.

[23] W. B. Muse and K. T. Phelps, Orthogonal quadruple systems and 3-frames. J. Combin. Theory A 104 (2003) 115-124.

[24] R. G. Stanton and R. C. Mullin, Some new results on the covering number $N(t, k, v)$. Combinatorial Mathematics VII (Lecture Notes in Math. 829, Springer, Berlin 1980). 51-58.

[25] L. Teirlinck, Some new 2-resolvable Steiner quadruple systems, Design Codes Cryptogr. 4 (1994) 5-10. 